\title{
The potential for imaging low-mass companions to solar-type stars: Utilising radial-velocity data from the AAPS, Keck \& Lick projects
}

\author{
J. S. Jenkins ${ }^{1}$, H. R. A. Jones ${ }^{1}$, C. G. Tinney ${ }^{2}$, C. McCarthy ${ }^{3}$, \\ B. D. Carter ${ }^{4}$, A. J. Penny ${ }^{5}$ \& D. J. Pinfield ${ }^{1}$ \\ ${ }^{1}$ University of Hertfordshire, College Lane, Hatfield, Hertfordshire, AL10 9AB \\ ${ }^{2}$ Anglo-Australian Observatory, PO Box 296, Epping, NSW 1710, Australia \\ ${ }^{3}$ Department of Physics and Astronomy, San Francisco State University, San Francisco, \\ CA 94132 \\ ${ }^{4}$ Faculty of Sciences, University of Southern Queensland, Toowoomba, 4350, Australia \\ ${ }^{5}$ Harvard-Smithsonian Center for Astrophysics, 60 Garden St, MA 02138,USA
}

\begin{abstract}
We are about to embark on a project to directly image low-mass companions orbiting within 1.5" of their host stars. Our long-term aim is to reach down into the planetary-mass regime. In order to start this project we have time on the VLT NAOS/CONICA (NACO) facility and in the near future we plan to make use of the Near-Infrared Coronographic Imager (NICI) instrument mounted on Gemini-South. Our targets are F-M type dwarfs, and have been selected from the Anglo-Australian Planet Search (AAPS), Keck and Lick search lists. These surveys have been periodically monitoring over 1200 stars between them for the past $\sim 8$ years with precisions typically around $3 \mathrm{~ms}^{-1}$. Our target list comprises objects which show significant evidence for companions on long-period orbits. Having a priori knowledge of companion characteristics allows us the advantage of prioritising our target list. Given the relatively high incidence of multiple planet systems found so far our search may also serve to provide useful constraints on any large separation, massive companions in our target systems.
\end{abstract}

Keywords. instrumentation: adaptive optics, instrumentation: high angular resolution, stars: low-mass, brown dwarfs; stars: planetary systems.

\section{Introduction}

The detection of over 150 planets orbiting sun-like stars has revolutionised our knowledge of our local neighbourhood and our position in it. Yet planets are not the sole close companions to solar-type stars. For instance, Duquennoy, Mayor \& Halbwachs (1991) discussed stellar multiplicity in a series of papers, focusing primarily on spectroscopic binaries (SB) found using the CORAVEL spectrometers. The bridge in mass-space between giant planets and SBs is huge, and it is here where cool objects such as Brown Dwarfs (BD) reside.

Radial-velocity (RV) surveys have revealed very few BDs orbiting solar-type stars (leading to the phrase 'brown dwarf desert' being coined to describe this paucity e.g. Marcy \& Butler (2000)) though beyond $\sim 4$ AU there are few RV data points. This arises because the RV method requires at least one full orbit to reliably constrain a companion's characteristics, which is a very time consuming process. Direct imaging techniques can be used in conjunction with RV projects to probe the extremities of these systems, without 
the need to wait for a long period of time, admittedly at the cost of performing a very technically challenging observation. Liu et al. (2002) used the Gemini-North and Keck Adaptive Optics (AO) systems to obtain three epochs of images of the BD companion to HR 7672, which had been indicated by the stellar RV signature. The flux ratio was found to be 8.6 magnitudes at a separation of 0.79 ". This level of contrast at this separation was pushing the limits of the instruments used. However the introduction of Simultaneous Differential Imaging (SDI) has allowed higher contrasts at smaller separations for the coolest stellar companions, e.g. $\Delta \mathrm{H} \sim 13$ at $\sim 0.5 "$ from Mugrauer \& Neuhauser (2005).

\section{Target Selection}

\subsection{Angular Separation}

Our target list is drawn from the AAPS, Keck and Lick planet search projects. The lowest mass companions, containing all our planetary-mass objects, have been taken from the compendium of Butler et al. (2006). The stars are FGKM-type stars, bright $(\mathrm{H}<7.7)$ and are accessible to the VLT and Gemini-South facilities $\left(\operatorname{dec}<+40^{\circ}\right)$. The other important characteristic these stars share is a long term radial-velocity profile consistent with an orbiting low-mass companion. These companions are inferred to have a wide range of masses $\left(1 \leqslant \mathrm{M} \sin i \leqslant 725 \mathrm{M}_{\mathrm{J}}\right)$, all masses below $1 \mathrm{M}_{\mathrm{J}}$ were fixed to 1 to fit in with the model limits. They also have accurate distances $(<110 \mathrm{pc})$, which were extracted from the Hipparcos (ESA, 1997) catalogue. It must be noted that unlike the Hipparcos data, the derived orbital properties are not well constrained, mostly because the companion orbits are much longer than the monitoring baselines of the surveys, and in some cases the companion properties have been derived with no inflection in the RV curve. The fit to these objects produce semimajor axis lower limits, which will most likely be much larger than the values we have derived. Also the eccentricity of most of the BDs and SBs are fixed to zero, causing more separation ambiguity.

\subsection{Contrast Ratio}

We found JHK magnitudes for the primary stars in the 2MASS catalogue (http://irsa.ipac.caltech.edu/) and using the Hipparcos distances we were able to generate accurate absolute JHK magnitudes. For the absolute magnitudes of the companions however, we used the non-grey evolutionary tracks of Burrows et al. (1997) and the NextGen models of Allard et al. (1997). We assumed ages from Valenti \& Fischer (2005) and minimum masses from our Keplerian fits. We split the companions into two groups depending on which model we could use to generate robust magnitudes. The split was made on the basis of companion effective temperatures, with all the objects using the Burrows et al. models having an $\mathrm{T}_{\text {eff }}<2000 \mathrm{~K}\left(\mathrm{M} \sin i<85 \mathrm{M}_{\mathrm{J}}\right)$ and all other objects using the NextGen models. A custom spline fitting procedure obtained the bolometric luminosities for all our companions. The model split between the cooler and hotter companions is clearly apparent. We have 156 companions below the strong methane absorption boundary $(1200 \mathrm{~K})$ (shown by the dot-dashed vertical line on the plot), apart from their too low luminosity we expect them to be good SDI targets. There are also 23 companions with effective temperatures above 1200K, 22 of which have $\mathrm{T}_{\text {eff }}>2000 \mathrm{~K}$ and are represented by triangles in Fig. 2 .

A major problem faced when attempting to image close-in companions to bright stars, is the contrast difference. To work out the contrasts between the stars and their companions we had to obtain the JHK magnitudes of the companions, both from internal and reflected flux. To accomplish this we used the median colours and bolometric corrections (BC) from Leggett et al. $(2002)\left(\mathrm{BC}_{\mathrm{T}}=2.06 ; \mathrm{BC}_{\mathrm{L}}=3.25\right.$, the subscripts denote 


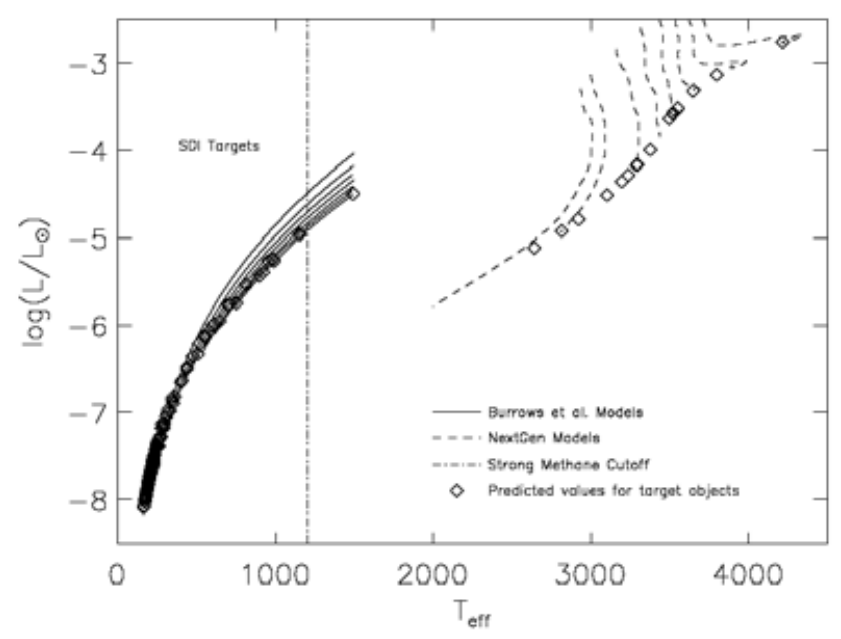

Figure 1. The solid lines are Burrows et al. (1997) models for different masses and ages. The dashed lines represent a range of masses and ages for the NextGen models of Allard et al. (1997), which is populated by our high-mass targets. The dot-dashed line marks the turn-off of strong methane absorption in cool dwarfs and provides a useful boundary for SDI targets. The discontinuity between the models is the LT boundary region, which is not very well modelled, thus the Burrows et al. models were truncated.

spectral type). This correction gave the absolute $\mathrm{K}$ magnitudes and, using the colours $\left(\mathrm{H}-\mathrm{K}_{\mathrm{T}}=-0.04 ; \mathrm{H}-\mathrm{K}_{\mathrm{L}}=0.70\right)$, we were able to generate their expected absolute $\mathrm{H}$-band magnitudes. For all planetary-mass companions we assumed Jupiter characteristics. We then simplistically simulated the expected reflected flux in the H-band and added this component to the internal flux. This was done by using simple geometry and assuming Jupiter's radius and albedo. The total absolute magnitudes of the companions were then subtracted from the absolute magnitudes derived for the primaries giving the contrast $\Delta$ H. Fig. 2 shows the contrasts and separations of all the objects we propose to image. The objects represented by filled circles are all companions with strong methane absorption. The bulk of the objects lie within 0.3 " of the primaries due to the bias introduced by the short RV baseline. The majority of these lower-mass objects ( $\mathrm{M} \sin i<15 \mathrm{M}_{\mathrm{J}}$ ) also have high contrasts $(\Delta \mathrm{H}>10)$, putting them below the NACO-SDI threshold. However, two objects have larger separations $>0.3$ ", approaching the separations of the already discovered objects of McCaughrean et al. (2003), Chauvin et al. (2004) and Neuhauser et al. (2005) and, combined with a $\Delta \mathrm{H}<13.5$, they could be amenable to SDI imaging. The NACO-SDI (Mugrauer \& Neuhauser (2005)) and NICI (Gemini (2005)) sensitivities are highlighted on Fig. 2 by dotted and dashed lines respectively. Once the masses and semimajor axes are more precisely defined, the companion magnitudes and separations will most likely increase giving lower contrasts and more viable targets. This has been highlighted on the plot by arrows on one of the objects near to current detection thresholds. The arrows represent the direction in which all objects are expected to move once inclination and eccentricity effects are considered and more RV data points acquired. Another major source of uncertainty is age. For example, the (1-sigma) age uncertainty of the solid circle companion with arrows plotted is $\pm 2.1 \mathrm{Gyr}$, giving rise to a \pm 2.1 mag error in $\Delta \mathrm{H}$ with the primary. All companions with $\mathrm{T}_{\text {eff }}>2000 \mathrm{~K}$ (triangles in Fig. 2) have H-band magnitudes less than 15, allowing direct imaging using normal AO 


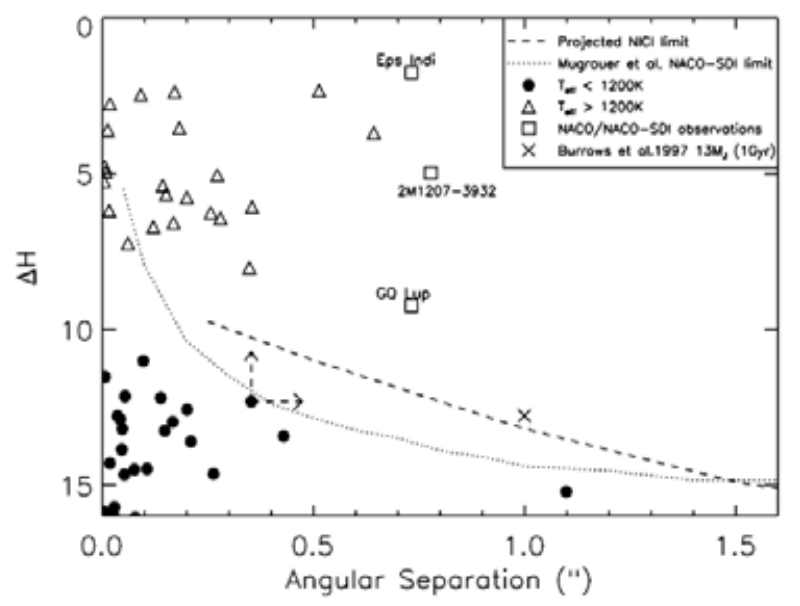

Figure 2. Triangles represent targets with $\mathrm{T}_{\text {eff }}>1200 \mathrm{~K}$, filled circles are targets $<1200 \mathrm{~K}$ (amenable to SDI) and the squares are companions to young stars already found by NACO \& SDI. For comparison, the dotted line shows the NACO-SDI sensitivity (Mugrauer \& Neuhauser (2005)) and the dashed line shows the NICI sensitivity (Gemini (2005)). The arrows show the most probable shift direction for one of the objects once better RV parameters have been derived and inclination and eccentricity effects considered. The single cross represents a $13 \mathrm{M}_{\mathrm{J}}$ object at $5 \mathrm{AU}$ orbiting a G5V star with an age of 1 Gyr and a distance of 5pc.

techniques. Four of these objects have separations larger than 0.35 " and $\Delta H$ less than 8, making excellent targets. All planetary-mass companions are off the Fig. 2 plot scale. The cross in Fig. 2 shows the position of a $13 \mathrm{M}_{\mathrm{J}}$ planet in a Jupiter-like orbit as a companion to a $1 \mathrm{Gyr}$, Sun-like star at 5pc. This type of object resides extremely close to the plotted instrument thresholds, highlighting just how difficult it is to obtain a direct image of any planetary-mass object with the current suite of instruments available. However, RV studies have revealed a high number of multiple planet systems, therefore imaging planet-host stars may provide useful constraints on any longer period, massive companions not yet revealed in the RV dataset.

\section{References}

Allard, F., et al. 1997, ARA\&A 35, 137

Blundell, J., et al., MNRAS submitted

Burrows, A., et al. 1997, SPIE 491, 856

Butler, R.P., et al. 2006, ApJ in prep

Chauvin, G., et al. 2004, A\&A 425, L29

Duquennoy, A., Mayor, M., \& Halbwachs, J.L. 1991, A\& A 88, 281

Gemini 2005, http://www.gemini.edu/sciops/instruments/nici/niciCampaign.html

Leggett, S.K., et al. 2002, ApJ 564, 452

Liu, M.C., et al 2002, ApJ 571, 519

Marcy, G.W. \& Butler, R.P. 2000, PASP 112, 137

McCaughrean, M.J., et al. 2003, A\&A 413, 1029

Mugrauer, M. \& Neuhauser, R. 2005, MNRAS 361, L15

Neuhauser, R., et al. 2005, A\&A 435, L13

Valenti, J. \& Fischer, D. 2005, ApJS 159, 141 